\title{
Bimodality of the viscoelastic response of a dense liquid and comparison with the frictional responses at short times
}

\author{
Sarika Bhattacharyya \\ Solid State and Structural Chemistry Unit, Indian Institute of Science, Bangalore 560 012, India \\ Biman Bagchia) \\ Solid State and Structural Chemistry Unit, Indian Institute of Science, Bangalore 560 012, India \\ and Jawaharlal Nehru Centre for Advanced Scientific Research, Jakkur, Bangalore 560 012, India
}

\begin{abstract}
While the time dependence of the friction on a tagged particle in a dense liquid has been investigated in great detail, a similar analysis for the viscosity of the medium and the interrelationship between the two has not been carried out. This is despite the close relation always assumed, both in theoretical and experimental studies, between friction and viscosity. In this article a detailed study of the time and frequency dependencies of the viscosity has been carried out and compared with those of the friction. The analysis is fully microscopic and is based on the mode coupling theory (MCT). It is found that for an argonlike liquid near its triple point, the initial decay of the viscosity occurs with a time constant of the order of $100 \mathrm{fs}$, which is close to that of the friction. The frequency dependent viscosity shows a pronounced bimodality with a sharp peak at the low frequency and a broad peak at the high frequency; the usually employed Maxwell's relation fails to describe the peak at the high frequency. A surprising result of the present study is that both the bare and the MCT values of viscosity and friction individually sustain a ratio which is close to the value predicted by the Stokes relation, even when Navier-Stokes hydrodynamics itself seems to have little validity.
\end{abstract}

\section{INTRODUCTION}

The well-known Stokes relation is often used to connect the friction $(\zeta)$ on a spherical molecule with the viscosity $(\eta)$ of the medium. This connection goes beyond the ordinary Stokes relation; even the generalized hydrodynamics describes the frequency $(\omega)$ dependent friction in terms of frequency dependent viscosity. ${ }^{1}$ While the hydrodynamic theory always predicts this near equivalence of the friction and the viscosity, microscopic theories seem to provide a drastically different picture. $^{2}$ In the mode coupling theory, the friction on a tagged molecule is expressed in terms of contributions from the density and the transverse current modes. The latter is expressed in terms of viscosity. Previous studies have shown that although for solutes with size much larger than the solvent it is this current mode which primarily determines the friction, in a neat liquid the friction coefficient is determined not by the transverse current mode but rather by the collision and the density fluctuation terms. ${ }^{2}$ Thus for neat liquids there is no a priori reason for such an intimate relation between friction and viscosity to hold.

Mode coupling theory provides the following rationale for the known validity of the Stokes relation between the zero frequency friction and the viscosity. According to MCT, both these quantities are primarily determined by the static and dynamic structure factors of the solvent. Hence both vary similarly with density and temperature. This calls into question the justification of the use of the generalized hydro-

${ }^{a)}$ Electronic mail: bbagchi@sscu.iisc.ernet.in dynamics for molecular processes. The question gathers further relevance from the fact that the time $(t)$ correlation function determining friction (the force-force) and that determining viscosity (the stress-stress) are microscopically different.

In this article we are concerned with the problems that can be articulated in terms of the following questions. What is the relation between $\eta(t)$ and $\zeta(t)$ at short times? Does the ratio between the two retain a Stokes-like value at all times? And how does the relation behave as a function of frequency?

A further motivation of the present article comes from the the following observations. Many chemical dynamical processes, such as nonpolar solvation dynamics, ${ }^{3}$ can be described in terms of the frequency dependent viscosity. Viscoelastic responses are required to understand the processes involving the rate of change in shape or size of molecules in liquids. ${ }^{4}$ Note that it is the frequency dependent viscosity which is readily accessible experimentally, whereas the frequency dependent friction is a purely theoretical entity. Another place where a knowledge of this interrelationship between $\zeta(\omega)$ and $\eta(\omega)$ is required is in understanding the viscosity dependence of activated processes in viscous liquids; this is a subject of much current interest. ${ }^{5}$ In the elegant Zwanzig-Bixon calculation ${ }^{1}$ of the frequency dependent friction, the frequency dependence of the viscosity was assumed to be given by the following Maxwell relation,

$$
\eta(\omega)=\frac{\eta}{1+i \omega \tau_{s}}
$$


where $\tau_{s}$ is the viscoelastic relaxation time, given by $\tau_{s}$ $=\eta / G_{\infty}$, where $G_{\infty}$ is the infinite frequency shear modulus and $\eta=\eta(\omega=0)$. From the above expression it is clear that this model assumes only one time scale. On the other hand, recent experimental studies ${ }^{6,7}$ have amply demonstrated that the solvent response is bimodal with at least two widely different time scales describing the response. ${ }^{8}$

In this paper a comparative study is done between the time/frequency dependence of the friction and the viscosity. It is found that if one includes only the binary interaction in the calculation of the time scale of the short-time dynamics, both viscosity and friction exhibit nearly the same time scale. When the triplet dynamics is included, both the responses become slower, with the viscosity being affected more than the friction. The time scale of both the responses are of the order of $100 \mathrm{fs}$. It is shown that the frequency dependent viscosity exhibits a clear bimodal dynamics, similar to that of the friction. ${ }^{8}$

We have also investigated the validity of the Stokes relation from the microscopic point of view and found the following surprising result. Individually and separately, the ratio of both the bare (binary dominated) and the mode coupling contributions to the friction and the viscosity follows a Stokes-like relation. Contrary to the hydrodynamic picture, we find that in the case of neat liquids in high density, it is more appropriate to think of the viscosity as being controlled by the diffusion or the friction. This is because in this regime the viscosity is primarily determined by the structural relaxation of the surrounding liquid, which in turn is determined by the diffusion.

The organization of the rest of the paper is as follows. Section II deals with the theoretical formulation and contains the mode coupling theory expressions for both the viscosity and the friction. Section III contains the numerical results. The validity of the Stokes relation is discussed in Sec. IV, and finally Sec. V concludes with a brief discussion on the results.

\section{THEORETICAL FORMULATION}

Let us consider a single tagged solute particle of the same size as the solvent molecules in a dense liquid. Let $v(r)$ denote the Lennard-Jones (LJ) interaction pair potential. Let $\sigma$ be the radius of the solute and the solvent molecules. The liquid is characterized by its number density $\rho$ and absolute temperature $T$. We shall use the reduced density $\rho^{*}=\rho \sigma^{3}$ as a measure of the density of the liquid and the reduced temperature $T^{*}=k_{B} T / \epsilon$ as the measure of the temperature. Here $k_{B}$ is the Boltzmann constant and $\epsilon$ the well depth of the LJ potential.

\section{A. Calculation of viscosity}

The time dependent shear viscosity is expressed in terms of the stress autocorrelation function and is given by

$$
\eta(t)=\left(V k_{B} T\right)^{-1}\left\langle J^{x y}(0) J^{x y}(t)\right\rangle,
$$

where $J^{x y}$ is the off-diagonal element of the stress tensor, and is given by

$$
J^{x y}=\sum_{j=1}^{N}\left[\left(p_{j}^{x} p_{j}^{y} / m\right)+F_{j}^{x} y_{j}\right] .
$$

Here $F_{j}^{x}$ is the $x$-component of the force acting on the $j$ th molecule, $p_{j}^{x}$ is the $x$-component of the momentum of the $j$ th molecule, and the corresponding position is $x_{j} . m$ is the mass of the same molecule.

The high-frequency shear modulus is given by

$$
G_{\infty}=\left(V k_{B} T\right)^{-1}\left\langle\left(J^{x y}(0)\right)^{2}\right\rangle .
$$

After a few steps of algebra, Eq. (4) can be reduced to the following exact expression: ${ }^{9}$

$$
G_{\infty}=\rho k_{B} T+\frac{2 \pi}{15} \rho^{2} \int_{0}^{\infty} d r g(r) \frac{d}{d r}\left[r^{4} \frac{d v(r)}{d r}\right],
$$

where $g(r)$ is the radial distribution function of the liquid.

By invoking the separation of time scales between the initial fast and the later slow decay, the time dependent viscosity of a liquid can be written as the sum of two different terms. The initial fast part arises due to the dynamics within the cage formed by the surrounding molecules and is expressed in terms of the static correlations. The fast part is followed by a slow long-time part which arises from the dynamical correlations and basically describes the relaxation of the cage due to the presence of the hydrodynamic modes like the density and the current. As discussed at length by Geszti, in dense liquids it is the density mode which primarily contributes to the long-time viscosity. ${ }^{10}$ The time dependent viscosity can thus be written as

$$
\eta(t)=\eta_{B}(t)+\eta_{\rho \rho}(t) .
$$

In the above expression, $\eta_{B}(t)$ is the short-time part which arises from the static correlations and $\eta_{\rho \rho}(t)$ is the long-time part which arises from the density mode contribution.

We first describe the calculation of $\eta_{B}(t)$. As only even powers of $t$ appear in $\eta(t), \eta_{B}(t)$ is approximated to be expressed in terms of a Gaussian function and written as

$$
\eta_{B}(t)=G_{\infty} \exp \left(-t^{2} / \tau_{\eta}^{2}\right) .
$$

In the above equation, $\tau_{\eta}$ is determined from the second derivative of $\eta(t)$.

$$
\tau_{\eta}=\sqrt{\frac{-2 G_{\infty}}{\ddot{\eta}(t=0)}} .
$$

In the liquid range, $\eta(t)$ is dominated purely by its potential part and thus the expression of viscosity reduces to

$$
\eta(t)=\left(V k_{B} T\right)^{-1}\left\langle\Sigma_{i} F_{i}^{x} y_{i} \Sigma_{j} F_{j}^{x}(t) y_{j}(t)\right\rangle .
$$

Next, one expresses the force in terms of derivative of the pair potential. One needs to include all the two- and three-particle contributions to obtain the proper short-time relaxation. The final expression for $\ddot{\eta}(t=0)$ is given by ${ }^{11}$

$$
\begin{aligned}
\ddot{\eta}(t=0)= & -\frac{4 \pi \rho^{2}}{15 m} \int_{0}^{\infty} d r r^{2}\left[r^{2}\left(v^{\prime \prime}\right)^{2}+2 r v^{\prime} v^{\prime \prime}\right. \\
& \left.+7\left(v^{\prime}\right)^{2}\right] g(r)-\frac{8 \rho^{2}}{75 m} \int_{0}^{\infty} d q q^{2}[S(q)-1] \\
& \times\left[2 A^{2}(q)+3 B^{2}(q)\right] .
\end{aligned}
$$

The functions $A(q)$ and $B(q)$ are defined by the following integrals: 
TABLE I. The calculated values of the friction $(\zeta)$ and the viscosity $(\eta)$ for four different thermodynamic states which are characterized by the reduced density, $\rho^{*}\left(=N \sigma^{3} / V, \sigma\right.$ is molecular diameter) and reduced temperature, $T^{*}$ ( $=k_{B} T / \epsilon, \epsilon$ is the Lennard-Jones energy parameter). The scaling of friction and viscosity are indicated on the table. The values of the ratio of the friction to viscosity are given in the last column of the table.

\begin{tabular}{llcll}
\hline \hline$\rho^{*}$ & $T^{*}$ & $\zeta / \sqrt{\epsilon / m \sigma^{2}}$ & $\eta \sigma^{2} / \sqrt{m \epsilon}$ & $m \zeta / \eta \mathscr{B}$ \\
\hline 0.6 & 2.0 & 8.61 & 0.63 & 27.29 \\
0.7 & 1.5 & 11.88 & 1.081 & 21.97 \\
0.844 & 0.728 & 29.44 & 3.20 & 18.37 \\
0.95 & 0.8 & 67.233 & 7.213 & 18.64 \\
\hline \hline
\end{tabular}

$$
\begin{aligned}
& A(q)=\int_{0}^{\infty} d r r^{2}\left[r v^{\prime \prime}+4 v^{\prime}\right] j_{1}(q r) g(r), \\
& B(q)=\int_{0}^{\infty} d r r^{2}\left[r v^{\prime \prime}-v^{\prime}\right] j_{3}(q r) g(r),
\end{aligned}
$$

where $j_{l}(x)$ are the spherical Bessel functions. $v^{\prime}$ $=d v(r) / d r$ and $v^{\prime \prime}=d^{2} v(r) / d r^{2} . S(q)$ is the static structure factor.

Note that the only approximation made in the derivation of Eq. (10) is the use of the Kirkwood superposition approximation for the triplet distribution function of the liquid. ${ }^{12}$ In a dense liquid at low temperature (near its triple point), this is not a bad approximation. ${ }^{12}$ But it does introduce an error in the short time which may even be as large as $50 \%$, for less dense liquids. Fortunately, this translates only to about an error of $10 \%$ maximum for the total viscosity. Actually, we find that at the triple point of argon the calculated value (in the usual scaled unit, see Table I) of viscosity is equal to 3.2 , while the simulated values lie between 2.9 and 3.85 and the experimental value is $3.0 .^{13}$

Next, we describe the calculation of the collective part of the viscosity, $\eta_{\rho \rho}(t)$, which, as mentioned earlier, arises from the density mode contribution. This is the long-time part of the viscosity and has the following mode coupling expression: ${ }^{10,11}$

$$
\begin{aligned}
\eta_{\rho \rho}(t)= & k_{B} T / 60 \pi^{2} \int_{0}^{\infty} d q q^{4}\left[S^{\prime}(q) / S(q)\right]^{2} \\
& \times\left[(F(q, t) / S(q))^{2}-\left(F_{B}(q, t) / S(q)\right)^{2}\right],
\end{aligned}
$$

where $S^{\prime}(q)$ is the first derivative of the static structure factor. $F_{B}(q, t)$ is the inertial part of the intermediate scattering function and is given by, $F_{B}(q, t)=\exp \left(-k_{B} T q^{2} t^{2} / 2 m S(q)\right)$. $F(q, t)$ is the intermediate scattering factor of the solvent. It is obtained through the Laplace transformation of the dynamic structure factor, $F(q, z)$, where $F(q, z)$ is expressed in terms of the well-known Mori continued-fraction expansion with its truncation at the second order. Thus $F(q, t)$ is given by $8,14,15$

$$
F(q, t)=\mathscr{B}^{-1} \frac{S(q)}{z+\frac{\left\langle\omega_{q}^{2}\right\rangle}{z+\frac{\Delta_{q}}{z+\tau_{q}^{-1}}} .}
$$

The Fourier frequency dependent viscosity is obtained by taking a Fourier transform of Eq. (6), and is given by

$$
\eta(\omega)=\eta_{B}(\omega)+\eta_{\rho \rho}(\omega) .
$$

The calculated values of the viscosity are found to be in good agreement with the simulated values for Lennard-Jones fluid. The agreement for the state points studied is within $5 \%$.

\section{B. Calculation of friction}

Just as in the case of viscosity, the separation of time scales between the binary collision and the repeated recollisions are invoked to decompose the friction on a tagged particle into a short-time and a long-time part. ${ }^{2,16}$ The resulting expression is given by

$$
\zeta(z)=\zeta_{B}(z)+\zeta_{R}(z)
$$

where $\zeta_{B}(z)$ is the binary part of the friction and $\zeta_{R}(z)$ is the long-time part which arises due to the correlated recollision of the solute particle with the solvent particles. The correlated recollision part is obtained by expanding the total friction in the basis set of the eigenfunctions of the Liouville operator. For a solute of size that is different from the solvent molecules, this contribution to the friction can be shown to be given by the following expression: ${ }^{2,16}$

$$
\zeta_{R}(z)=R_{\rho \rho}(z)-\left[\zeta_{B}(z)+R_{\rho \rho}(z)\right] R_{T T}(z) \zeta(z) .
$$

In the above expression, $R_{\rho \rho}(z)$ gives the coupling of the solute motion to the density modes of the solvent through the two-particle direct correlation function. $R_{T T}(z)$ gives the coupling to the transverse current through the transverse vertex function. $R_{\rho \rho}(z)$ and $R_{T T}(z)$ are obtained through Laplace transformation of $R_{\rho \rho}(t)$ and $R_{T T}(t)$, respectively. The expressions for $R_{\rho \rho}(t)$ and $R_{T T}(t)$ are given by ${ }^{2}$

$$
\begin{aligned}
R_{\rho \rho}(t)= & \frac{\rho k_{B} T}{m} \int\left[d \mathbf{q}^{\prime} /(2 \pi)^{3}\right]\left(\hat{q} \cdot \hat{q}^{\prime}\right)^{2} q^{\prime 2} \\
& \times\left[c_{12}\left(q^{\prime}\right)\right]^{2}\left[F^{s}\left(q^{\prime}, t\right)-F^{o}\left(q^{\prime}, t\right)\right] \\
R_{T T}(t)= & \frac{1}{\rho} \int\left[d \mathbf{q}^{\prime} /(2 \pi)^{3}\right]\left[1-\left(\hat{q} \cdot \hat{q}^{\prime}\right)^{2}\right] \\
& \times\left[\gamma_{d 12}^{t}\left(q^{\prime}\right)\right]^{2} \omega_{o 12}^{-4}\left[F^{s}\left(q^{\prime}, t\right)\right. \\
& \left.-F^{o}\left(q^{\prime}, t\right)\right] C_{t t}\left(q^{\prime}, t\right) .
\end{aligned}
$$$$
\times\left[c_{12}\left(q^{\prime}\right)\right]^{2}\left[F^{s}\left(q^{\prime}, t\right)-F^{o}\left(q^{\prime}, t\right)\right] F\left(q^{\prime}, t\right),
$$

The input parameters required to calculate $R_{\rho \rho}(t)$ are the two-particle direct correlation function between the solute and the solvent, $c_{12}(q)$, the dynamic structure factor of the solute, $F^{s}(q, t)$, the inertial part of the dynamic structure factor of the solute, $F^{o}(q, t)$, and the dynamic structure factor of the solvent, $F(q, t)$. Note here that the product $c_{12}(q) F(q, t)$ in Eq. (8) represents the modified dynamic structure factor of the solvent around the solute. Hence the fact that the structure of the solvent is different around the solute from that in the bulk has been included in Eq. (18). The input parameter necessary for the calculation of $R_{T T}(t)$ is the vertex function of the solute-solvent mixture, $\gamma_{d 12}^{t}(q)$, which actually takes care of the interaction of the solute motion with the current mode of the solvent. The other param- 
eters required are the Einstein frequency of the solute in presence of the solvent molecules, $\omega_{o 12}$, the dynamic structure factor of the solute, and the transverse current autocorrelation function of the solvent, $C_{t t}(q, t)$.

Next we describe the calculation of the binary part $\zeta_{B}(t)$, which is controlled by the short-time dynamics. For a continuous potential, the calculation of $\zeta_{B}(t)$ is nontrivial. The steps involved in the calculation are outlined below. As only the even powers of $t$ appear in $\zeta_{B}(t)$, it can be written as

$$
\zeta_{B}(t)=\omega_{o 12}^{2} \exp \left(-t^{2} / \tau_{\zeta}^{2}\right)
$$

$\omega_{o 12}$ is the Einstein frequency of the solute in the presence of the solvent, ${ }^{2}$

$$
\omega_{o 12}^{2}=\frac{\rho}{3 m} \int d \mathbf{r} g_{12}(r) \nabla^{2} v(r),
$$

where $g_{12}(r)$ is the radial distribution function of the solutesolvent mixture. In Eq. (20), the relaxation time $\tau_{\zeta}$ is determined from the second derivative of $\zeta_{B}(t)$ at $t=0$ and is given exactly by ${ }^{16}$

$$
\begin{aligned}
\omega_{o 12}^{2} / \tau_{\zeta}^{2}= & \left(\rho / 3 m^{2}\right) \int d \mathbf{r}\left(\nabla^{\alpha} \nabla^{\beta} v(\mathbf{r})\right) g(\mathbf{r})\left(\nabla^{\alpha} \nabla^{\beta} v(\mathbf{r})\right) \\
& +(1 / 6 \rho) \int\left[d \mathbf{q} /(2 \pi)^{3}\right] \gamma_{d}^{\alpha \beta}(\mathbf{q}) \\
& \times(S(q)-1) \gamma_{d}^{\alpha \beta}(\mathbf{q}),
\end{aligned}
$$

where summation over repeated indices is implied. Here $S(q)$ is the static structure factor. The expression for $\gamma_{d}^{\alpha \beta}(\mathbf{q})$ is written as a combination of the distinct parts of the second moments of the longitudinal and transverse current correlation functions $\gamma_{d}^{l}(\mathbf{q})$ and $\gamma_{d}^{t}(\mathbf{q})$, respectively. Note that the use of the Kirkwood superposition approximation for the triplet correlation function has been used in deriving the second term of Eq. (22). For the friction, unlike for viscosity, the effects of the triplet term are small.

In order to solve all the above equations one thus needs to calculate a large number of dynamical variables. The expressions of these variables and the method of calculations are available elsewhere. ${ }^{2}$

Finally, note that the expression for the recollision friction given by Eq. (17) involves the full friction itself on the right-hand side. Thus the equations are to be solved selfconsistently. This is achieved by substituting the expression of $\zeta_{R}(z)$ in Eq. (16). The final expression for the total friction is now given by

$$
\frac{1}{\zeta(z)}=\frac{1}{\zeta_{B}(z)+R_{\rho \rho}(z)}+R_{T T}(z) .
$$

As discussed before, ${ }^{2}$ this expression goes over to the expression derived earlier by many, ${ }^{17-19}$ only if the contribution of the density mode is neglected and the hydrodynamic approximation of the current mode is employed. Equation (23) is a generalized expression where the microscopic term is renormalized by the density mode.

We shall need friction also as a function of the Fourier frequency $(\omega)$. In the notation followed, $(\omega)$ is related to the

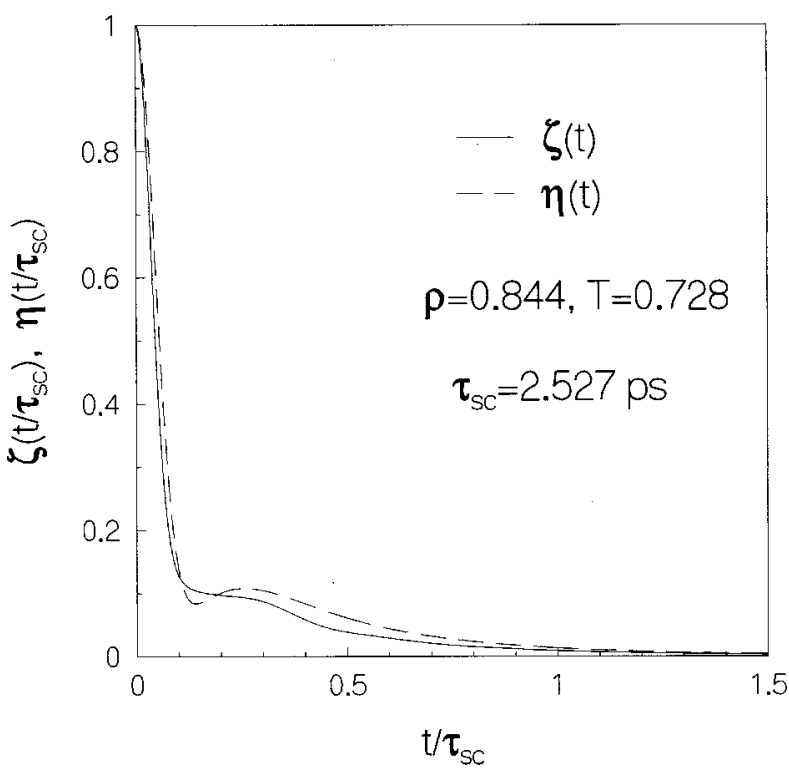

FIG. 1. The time dependence of the friction $\zeta(t)$ (solid line) and the viscosity $\eta(t)$ (dashed line), for a Lennard-Jones liquid near its triple point $\left(\rho^{*}=0.844\right.$ and $\left.T^{*}=0.728\right)$. The friction and the viscosity are normalized by their initial values to facilitate comparison of the dynamics. The time is scaled by the usual dimensionless time, $\tau_{s c}=\left(m \sigma^{2} / k_{B} T\right)^{0.5}$, which is here equal to 2.527 ps. For more details see the text.

Laplace frequency via $(\omega=i z)$. From the above expressions we have calculated $\zeta(z=0)$ self-consistently. Using this value of friction in Eqs. (18) and (19) we have calculated the Fourier frequency dependent total friction

$$
\zeta(\omega)=\zeta_{B}(\omega)+R_{\rho \rho}(\omega) .
$$

\section{NUMERICAL RESULTS}

The numerical calculation of the time dependent viscosity and friction requires a detailed knowledge of the radial distribution function and the wave number dependent twoparticle direct correlation function of the liquid. The radial distribution function can be calculated accurately by using the Zerrah-Hansen scheme. ${ }^{20}$ Although the Zerrah-Hansen scheme $^{20}$ is quite accurate in the real space, it is known to provide a less accurate description in the wave number space. Therefore, the wave number dependent direct correlation function is obtained by the following method. First, the effective hard-sphere diameter and density of the LennardJones system is obtained by using the well-known Weeks-Chandler-Anderson ${ }^{21}$ perturbation scheme. In the next step, the two-particle direct correlation function for the Lennard-Jones liquid is obtained from the Wertheim-Thiele solution of the Percus-Yevick equation using this effective hard-sphere diameter and density.

We have already discussed that the calculated value of the total zero frequency viscosity at the triple point is in very good agreement (within 10\%) with both the simulated and the experimental results. The calculated value of the diffusion coefficient at the triple point is $1.325 \times 10^{-5}$, while the simulated value is $1.75 \times 10^{-5} \mathrm{~cm}^{2} \mathrm{~s}^{-1} .15$

Figure 1 depicts a comparative study between the time dependent friction and the viscosity at $\rho^{*}=0.844$ and $T^{*}$ 


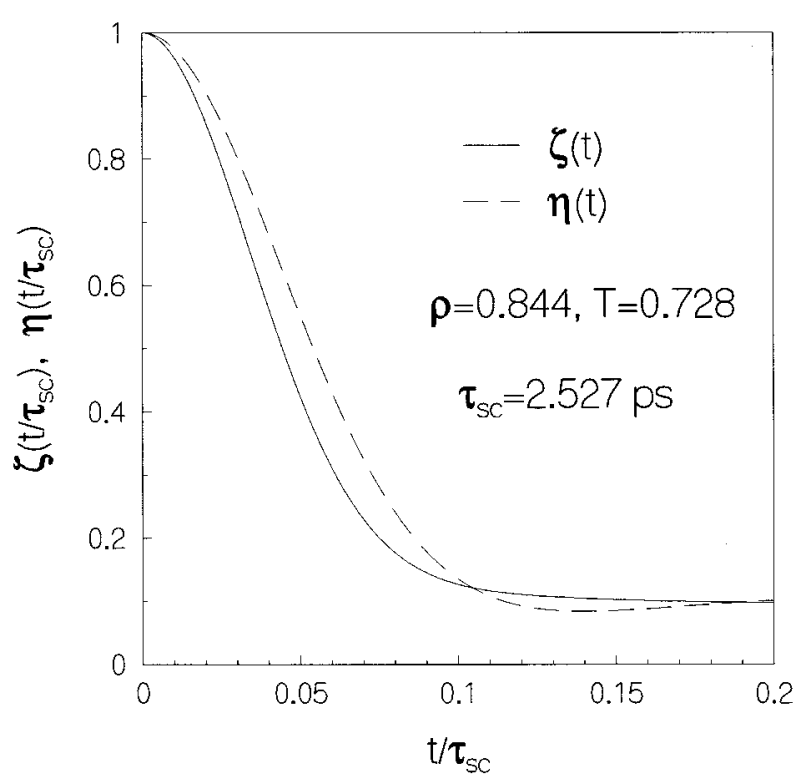

FIG. 2. The same as Fig. 1-the short-time dynamics is shown in an elongated ordinate scale to facilitate comparison.

$=0.728$. Thus, we are considering an argonlike liquid near its triple pont. In this figure, both the viscosity and the friction have been normalized to unity at $t=0$ by their respective initial values. This figure has several interesting features. Both the two quantities exhibit a pronounced ultrafast Gaussian decay which accounts for almost $90 \%$ of the total relaxation. The Gaussian time constants are equal to 130 for the friction and 160 fs for the viscosity. The second interesting aspect is that both the two quantities exhibit slow long-time decay which is also comparable.

It is worthwhile to discuss the relative contributions of the binary and the three-particle correlations to the initial decay. If the triplet correlation is neglected, then the values of the Gaussian time constants are equal to 89 and 93 fs for the friction and the viscosity, respectively. Thus, the triplet correlation slows down the decay of viscosity more than that of the friction. The greater effect of the triplet correlation is in accord with the more collective nature of the viscosity. This point also highlights the difference between viscosity and friction. As already discussed, the Kirkwood superposition approximation has been used for the triplet correlation function to keep the problem tractable. This introduces an error which, however, may not be very significant for an argonlike system at triple point. Figure 2 displays the similarity between the short-time dynamics of the friction and viscosity on an enlarged scale.

Figure 3 depicts the imaginary part of the frequency dependent viscosity which clearly demonstrates the bimodality of the viscoelastic response. In the same figure we have also plotted the prediction from the Maxwell's relation. In the latter we have calculated the relaxation time $\tau_{s}$ by the wellknown expression, $\tau_{s}=\eta(z=0) / G_{\infty}$. It shows that the Maxwell relation produces only one peak at low frequency and provides inadequate description at higher frequencies. Another important aspect of this graph is that the simple Max-

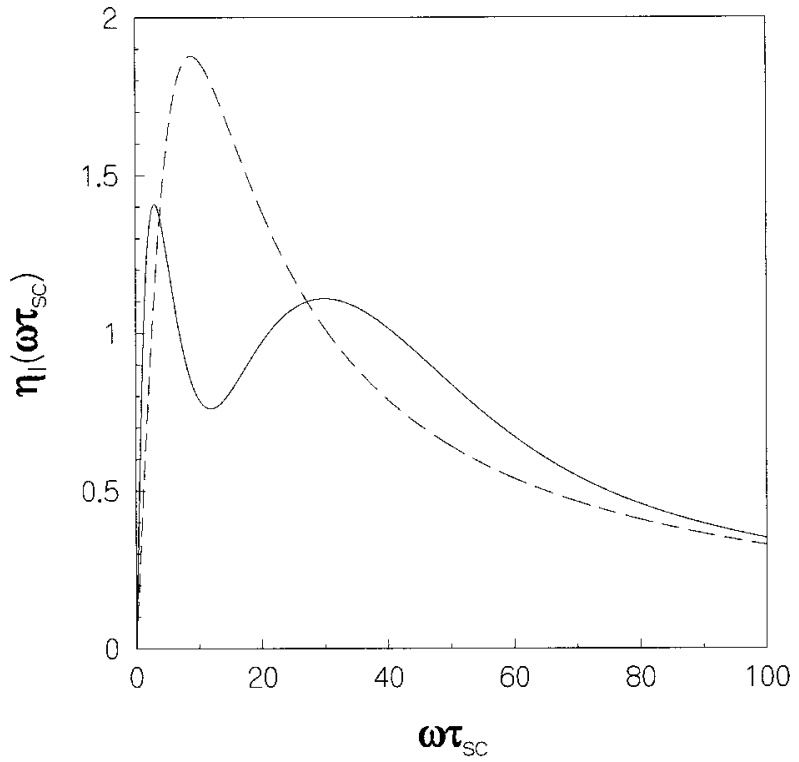

FIG. 3. The imaginary part of the calculated viscosity is plotted as a function of the frequency at the triple point (solid line). Also shown is the prediction of the Maxwell viscoelastic model (dashed line), given by Eq. (1) of the text. The viscosity is scaled by $\sigma^{2} / \sqrt{\left(m k_{B} T\right)}$ and the frequency is scaled by $\tau_{s c}^{-1}$, where $\tau_{s c}=\left[m \sigma^{2} / k_{B} T\right]^{1 / 2}=2.527 \mathrm{ps}$.

well relation fails to describe adequately even the low frequency peak.

In Fig. 4, the frequency dependent real part of the viscosity has been plotted. It should be pointed out that the frequency dependence of both the real and the imaginary part bear striking resemblance to those of the friction, discussed in Ref. 8.

Figure 5 shows the calculated ratio $m \zeta /(\eta \mathscr{R})$ against the calculated viscosity $\eta$, for a variation of $\eta$ for more than an order of magnitude-here $\mathscr{B}$ is the radius of an atom,

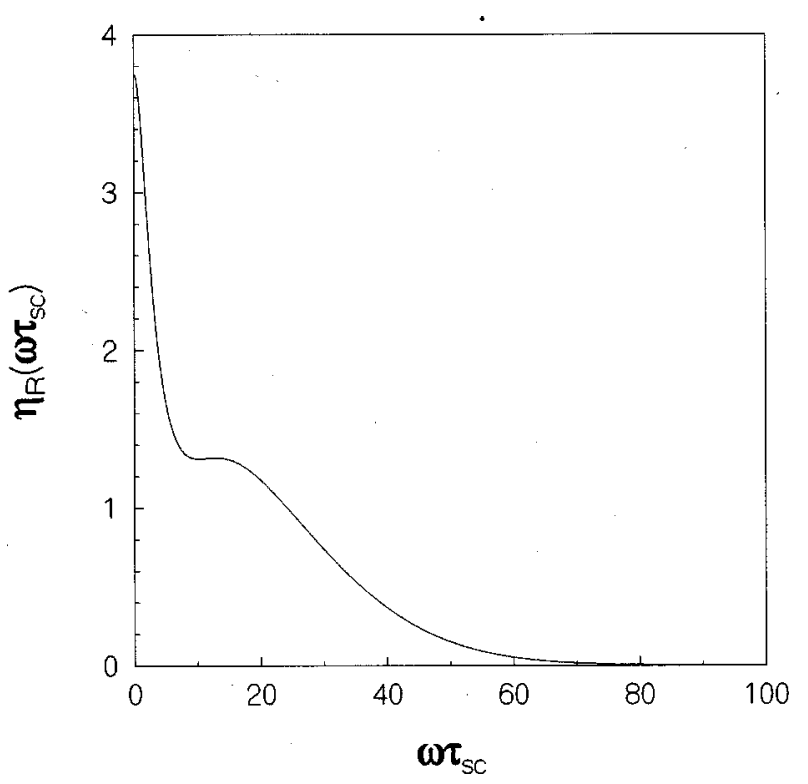

FIG. 4. The real part of the calculated viscosity is plotted as a function of the frequency at the reduced density $\rho^{*}=0.844$ and the reduced temperature $T^{*}\left(=k_{B} T / \epsilon\right)=0.728$. The viscosity is scaled by $\sigma^{2} / \sqrt{\left(m k_{B} T\right)}$ and the frequency is scaled by $\tau_{s c}^{-1}$, where $\tau_{s c}=\left[m \sigma^{2} / k_{B} T\right]^{1 / 2}=2.527 \mathrm{ps}$. 


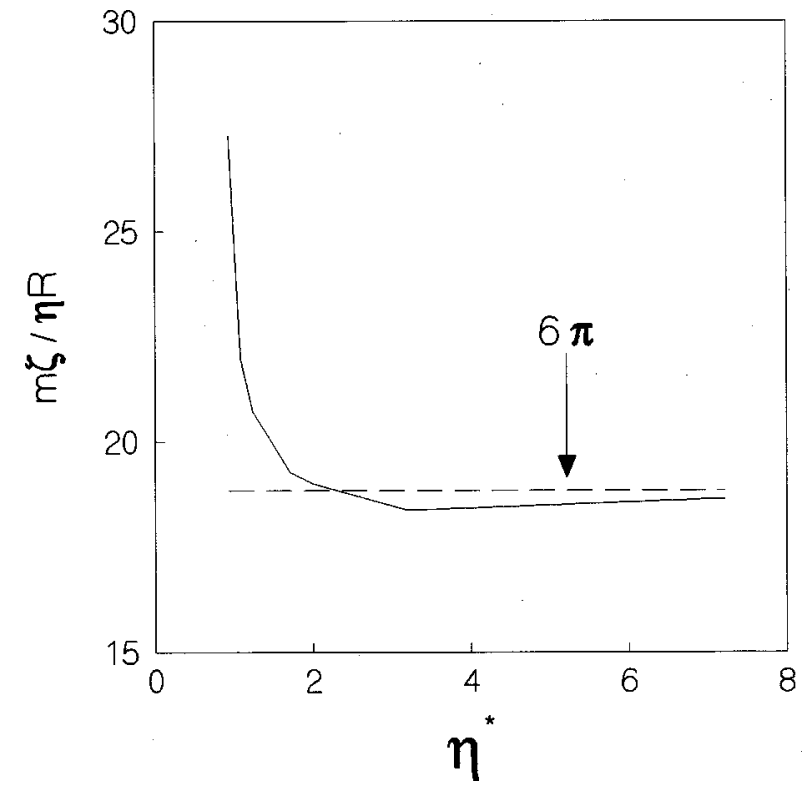

FIG. 5. The ratio of the calculated friction to the calculated viscosity is plotted against the reduced viscosity. These values are obtained at various densities and temperatures, all given in Table I. The viscosity is scaled by $\sigma^{2} / \sqrt{(m \epsilon)}$. The prediction of the Stokes relation with the stick boundary condition is shown by a dashed line.

$\mathscr{B}=\sigma / 2$. The values of $\rho^{*}, T^{*}, \eta$, and $\zeta$ are all given in Table I. It can be seen from this figure that in dense liquids the ratio $m \zeta /(\eta \mathscr{R})$ is close to $6 \pi$, which is indeed surprising. Any hydrodynamiclike behavior is not seen at low densities where the value of the viscosity decreases faster with lowering the density than the friction. Actually, such breakdown of hydrodynamic behavior has also been observed in the computer simulations of Lennard-Jones ellipsoids. ${ }^{22}$

\section{STOKES RELATION REVISITED}

In an interesting previous study, Mehaffey and Cukier ${ }^{19}$ showed that when the size of the solute becomes very large compared to the solvent molecules, a form akin to StokesEinstein relation is recovered. Earlier we showed in Ref. 2 that when the size of the diffusing particle becomes larger than the size of the solvent molecules, the contribution of the current mode [the second term in Eq. (23)] to the total diffusion can become larger than the combined contribution of the binary and the density modes. Numerical calculation shows that this crossover takes place when the solutesolvent size ratio becomes somewhat larger than 2 . If the solute-solvent interaction energy remains unchanged, then one finds that the friction numerically converges to a value given by $4 \pi \eta R$ - that is, the slip limit.

As emphasized in Sec. I, the hydrodynamic derivation (based on the contribution of the current mode alone ${ }^{23}$ ) of the relation between the friction and the viscosity has no validity in the case of neat liquids (where the tagged molecule has the same size as a solvent molecule). On the other hand, the experiments, ${ }^{24}$ computer simulations, ${ }^{25}$ and the MCT calculations presented here all show that the ratio of friction to viscosity almost always lies between $4 \pi$ and $6 \pi$ even for a neat liquid. It is, therefore, imperative to analyze the cause of apparent validity of the Stokes relation in greater depth.

To explain the validity of the Stokes-Einstein (SE) relation for solutes of the same size as the solvent at high density, Mehaffey and Cukier ${ }^{19}$ have suggested the following expression for the diffusion coefficient,

$$
D=D_{E}+\frac{k_{B} T}{c \eta R}+D_{L},
$$

where $D_{E}$ is the Enskog diffusion coefficient, the second term on the right is the contribution from the ring term, and $D_{L}$ is the contribution to diffusion from the longitudinal density mode. According to Mehaffey and Cukier, the longitudinal density mode contribution may cancel the Enskog contribution and the diffusion will be expressed by the SE relation.

The analysis presented in this article and several previous works $2,8,16,17$ has shown that the contribution from the longitudinal mode is not an additive term to the diffusion but to the friction! Hence such a cancellation is not possible.

In the following we present a semiquantitative argument on the recovery of the hydrodynamic boundary condition from microscopic considerations.

An analysis of the relevant integrals [in Eqs. (13) and (18)] shows that the dominant contribution of the density mode to the viscosity and the friction comes from intermediate length scale $(8 \geqslant k \sigma \geqslant 3)$. That is, more than $90 \%$ of the contribution comes from a region surrounding the sharp first peak of the static structure factor, that is, around $k \sigma$ $=2 \pi$. At these values of the wave number, the dynamic structure factor is well determined by the following simple mean field expression first used by de Gennes many years ago,

$$
F(q, t)=S(q) \exp \left(\frac{-D q_{m}^{2} t}{S\left(q_{m}\right)}\right) .
$$

Here $D$ is the self-diffusion coefficient which is determined self-consistently from Eq. (23) and then is used in the above expression to provide a correct intermediate scattering function to be used in the calculation of the viscosity.

Further simplification can be made by using a simple prescription for the wave number dependence of the structure factor, as shown by Balucani. ${ }^{26}$ The above prescription provides fairly accurate values for the zero frequency friction and the viscosity.

It is, therefore, clear from the above discussion that the collective contribution to the viscosity is dominated by the structural relaxation, which in turn is determined by the rate of diffusion. Thus, it is more appropriate to consider the viscosity of the medium as being determined by the diffusion. This is, of course, a matter of perspective.

There is, however, an even more interesting aspect. Using the results of Balucani, ${ }^{11}$ it can be shown that the initial value of the viscosity and the friction are related approximately by

$$
\frac{m \zeta(t=0)}{\eta(t=0) \mathscr{R}} \approx \frac{20}{\rho^{*}} .
$$


For $\rho^{*}=0.844$ and $T^{*}=0.72$, Eq. (27) gives a value of the ratio equal to 23.6966 .

Now, we have already found that the decay of the normalized viscosity is slightly slower than that of the friction, and the ratio of the time constants is 160/124. Thus, the contribution of the bare part to the zero frequency friction is equal to $23.6966 \times 124 / 160$, which is equal to 18.364 . Therefore, the ratio of the bare part of the zero frequency friction to that of viscosity is nearly identical to $6 \pi$. This we believe provides a microscopic explanation for the results shown in Fig. 5.

It is to be noted that in the above discussion, although the numerical values of the prefactor are close to $6 \pi$, it does not in any way imply the stick boundary condition. The above calculation is based only on microscopic considerations and is semiquantitative in nature. The main point here is that in the high density liquid regime, the ratio of the friction to the viscosity attains a constant value independent of the viscosity (density and temperature) and this value is numerically close to (but less than) $6 \pi$.

It is now interesting to discuss the simulated values of this ratio at high density. For an argon system near the triple point at $\rho=.021 \AA^{-3}$ and $T=86.5 \mathrm{~K}$, the ratio is $4.7 \pi$. At $\rho=.021 \AA^{-3}$ and $T=95 \mathrm{~K}$, the ratio is $5.1 \pi$. In computing the above ratios, the friction is obtained from the Einstein relation using the known value of diffusion coefficient. ${ }^{25}$ It is perhaps fair to allow an uncertainty of $5 \%-15 \%$ in the determination of this ratio both by theory or simulation.

\section{CONCLUSION}

Let us first summarize the main results of the study. First, it is shown that the short-time viscous and frictional responses in a neat liquid occur essentially on the same time scale. The time dependence of this response is largely Gaussian, which is followed by a slow long-time decay. This biphasic response is a hallmark of dynamical processes in dense liquid. This is clearly reflected in the imaginary part of the frequency dependent viscosity as shown in Fig. 3. The second important result is that the time scale of the initial decay is of the order of $100 \mathrm{fs}$, which is typically the time scale observed both in polar and nonpolar solvation dynamics. In the present case, the ultrafast dynamics originates clearly from the nearest-neighbor static correlation. The third important result is the demonstration that the apparent validity of a Stokes-like relation between friction and viscosity has its root both in statics and dynamics. While the initial values of these quantities primarily determine the ratio, the nearly identical dynamics sustains this ratio even in the frequency plane.

As discussed before, in this work we did not consider the recovery of the Stokes relation for large solutes. This has been discussed in Ref. 2, where it was shown that for the same solute-solvent interaction the friction is determined primarily by the current mode when the solute-solvent size ratio becomes somewhat larger than 2 . The numerical values of the calculated friction were found to converge to a value given by $4 \pi \eta R$ - that is the slip boundary condition. However, if the solute-solvent attractive interaction also increases with the size, a different picture might emerge. ${ }^{27}$
On the other hand, we show here that for solutes of the same size as the solvent, both the friction and the viscosity are determined by the microscopic terms and numerically the friction converges to a value close to (but less than) $6 \pi \eta R$. Thus the origin behind the validity of the Stokes relation for the same size solute is that both the microscopic friction and the viscosity are determined essentially by the same dynamical variables. We have emphasized that the occurrence of $6 \pi$ is not to be taken as a signature of the stick boundary condition.

The above discussion leads to the following important point. For a neat liquid the Navier-Stokes hydrodynamics cannot be used to justify apparent numerical validity of the Stokes relation. In this case, the validity of a Stokes-like relation between the viscosity and the friction can be explained only when the contributions from the bare (that is, the binary) and the density modes are both taken into account. Another point of interest is that while in hydrodynamics based analysis it is believed that it is the viscosity which determines the friction, the present study suggests that perhaps it is more meaningful to think in terms of the reverse scenario. In the region where hydrodynamics is not valid but a Stokes-like relation is obeyed, it is the diffusion (or the friction) which determines the viscosity and not vice versa.

Thus the recovery of the Stokes-Einstein relation for large solutes and for solutes of the same size as the solvent have completely different origins.

Note that although we have discussed diffusion and viscosity only in the normal liquid domain, the study can be extended to the region near the glass transition. The expressions for friction and viscosity will remain the same but the dynamical variables such as the dynamic structure factor will develop a long-time tail within a very narrow density range. ${ }^{28,29}$ This long-time tail of the dynamic structure factor accounts for the rapid rise of the value of viscosity and friction over this narrow density range. As discussed in Ref. 28, a decoupling between the diffusion and the viscosity can arise near the glass transition, which might lead to a complete breakdown of the SE relation in this regime. This decoupling might arise due to the existence of the slowly relaxing solidlike microdomains in the supercooled liquids. The presence of this solidlike domain gives rise to inhomogeneity in the liquid with some domains which are solidlike and others which are liquidlike. In each of these domains the SE law might be valid but the overall value of the diffusion can decouple from the measured viscosity.

The results presented here could also be used in various applications, for example in understanding nonpolar solvation and in activated barrier crossing dynamics. ${ }^{29}$

\section{ACKNOWLEDGMENTS}

We thank Mr. Ranjit Biswas for helpful discussions. This work was supported in part by grants from the Department of Science and Technology (India) and the Council of Scientific and Industrial Research (CSIR). S. Bhattacharyya thanks CSIR for a research fellowship.

\footnotetext{
${ }^{1}$ R. Zwanzig and M. Bixon, Phys. Rev. A 2, 2005 (1970).

${ }^{2}$ S. Bhattacharyya and B. Bagchi, J. Chem. Phys. 106, 1757 (1996).
} 
${ }^{3}$ M. Berg, J. Phys. Chem. A 102, 17 (1998).

${ }^{4}$ Y. Pomeau and P. Resibois, Phys. Rep. 19C, 63 (1975).

${ }^{5}$ R. M. Grote and J. T. Hynes, J. Chem. Phys. 73, 2715 (1980); J. T. Hynes, in The Theory of Chemical Reaction Dynamics, edited by M. Baer (CRC, Boca Raton, FL, 1985), Vol. IV, p. 171.

${ }^{6}$ M. Berg, Chem. Phys. Lett. 228, 317 (1994).

${ }^{7}$ T. Joo, Y. Jia, J-Y. Yu, M. J. Lang, and G. R. Fleming, J. Chem. Phys. 104, 6089 (1996); M. L. Horng, J. A. Gardecki, A. Papazyan, and M. Maroncelli, J. Phys. Chem. 99, 17,311 (1995).

${ }^{8}$ S. Bhattacharyya and B. Bagchi, J. Chem. Phys. 106, 7262 (1997).

${ }^{9}$ R. Zwanzig and R. D. Mountain, J. Chem. Phys. 43, 4464 (1965).

${ }^{10}$ T. Geszti, J. Phys. C 16, 5805 (1983).

${ }^{11}$ U. Balucani, Mol. Phys. 71, 123 (1990).

${ }^{12}$ J. P. Hansen and I. R. McDonald, Theory of Simple Liquids (Academic, New York, 1986).

${ }^{13}$ W. G. Hoover, D. J. Evans, R. B. Hickman, A. J. C. Ladd, W. T. Ashurst, and B. Moran, Phys. Rev. A 22, 1690 (1980).

${ }^{14}$ J. P. Boon and S. Yip, Molecular Hydrodynamics (McGraw-Hill International, New York, 1980).

${ }^{15} \mathrm{U}$. Balucani and M. Zoppi, Dynamics of the Liquid State (Clarendon, Oxford, 1994)

${ }^{16}$ L. Sjogren and A. Sjolander, J. Phys. C 12, 4369 (1979).
${ }^{17}$ J. T. Hynes, R. Kapral, and Weinberg, J. Chem. Phys. 70, 1456 (1979).

${ }^{18}$ T. Keyes, in Statistical Mechanics, Part B: Time Dependent Processes, edited by B. J. Berne (Plenum, New York, 1977).

${ }^{19}$ J. R. Mehaffey and R. I. Cukier, Phys. Rev. A 17, 1181 (1978).

${ }^{20}$ G. Zerrah and J. P. Hansen, J. Chem. Phys. 84, 2336 (1986).

${ }^{21}$ J. D. Weeks, D. Chandler, and H. C. Anderson, J. Chem. Phys. 54, 5237 (1971).

${ }^{22}$ S. Ravichandran, A. Perera, M. Moreau, B. Bagchi, J. Chem. Phys. 107, 8469 (1997).

${ }^{23}$ U. Balucani, R. Vallauri, and T. Gaskell, Ber. Bunsenges. Phys. Chem. 94, 261 (1990).

${ }^{24}$ B. H. C. Chen, C. K. J. Sun, and S. H. Chen, J. Chem. Phys. 82, 2052 (1985); R. Ravi and D. Ben-Amotz, Chem. Phys. 183, 385 (1994).

${ }^{25}$ See Ref. 15, Table 5.1, pg. 193 for the value of the diffusion coefficient; Table 6.1, pg. 261 for the viscosity value; pg. 264 by using the effective particle radius instead of the defined length scale " $a$."

${ }^{26}$ U. Balucani, R. Vallauri, T. Gaskell, and S. F. Duffy, J. Phys.: Condens. Matter 2, 5015 (1990).

${ }^{27}$ R. Biswas, S. Bhattacharyya, and B. Bagchi, J. Phys. Chem. B 102, 3252 (1998).

${ }^{28}$ S. Bhattacharyya and B. Bagchi, J. Chem. Phys. 107, 5852 (1997).

${ }^{29}$ R. Biswas and B. Bagchi, J. Chem. Phys. 105, 7543 (1996). 